\title{
Trial Secondary Objective
}

National Cancer Institute

\section{Source}

National Cancer Institute. Trial Secondary Objective. NCI Thesaurus. Code C85827.

The auxiliary purpose of the trial. 